\title{
Observational Cosmology
}

\section{François R. Bouchet*}

Institut d'Astrophysique de Paris, UMR7095, CNRS $\mathcal{G}$ Sorbonne Université, 75014 Paris, France

E-mail: bouchet@iap.fr

Despite the title, I am afraid that I will not be able to really do justice to the broad topic I was given to review. But I will show the current striking level of precise consistency between the CMB and LSS data within the $\Lambda C D M$ theoretical framework (with the acronyms CMB and LSS standing for Cosmic Microwave Background and Large Scale Structures). This part is largely based on the recent preprint "Planck 2018 results. I. Overview, and the cosmological legacy of Planck" where I had a chance to express my views at length on this topics :-).

Then after briefly recalling some of the currently open questions that cosmology will soon help address, as well as the tensions between some experimental results, I offer a short overview of experimental prospects which will insure that cosmology will remain as exciting in the coming decades as it has been in the last five!

European Physical Society Conference on High Energy Physics - EPS-HEP2019 -

10-17 July, 2019

Ghent, Belgium

${ }^{*}$ Speaker. 


\section{Introduction}

Observational cosmology is a striving field, with many ongoing experiments, and it is of course impossible to cover them all fairly within the confines of a single presentation. I therefore offer only a bird's eye view of selected topics, focusing on some of the more striking aspects for the future of the field.

The most precise and powerful determination of the cosmological model is currently offered by the results of the Planck satellite mission, with rather significant differences with respect to the previous results from the WMAP satellite [1]. Sketched out in 1992, selected by ESA in 1996, and launched in 2009, the Planck satellite ${ }^{1}$ [2, 3] was shut off in 2013, after collecting data that exceeded all expectations. Our third and final data release happened mid-2018, and the accompanying papers are now all in press. In short, Planck main scientific goal was to map the primordial CMB anisotropies to the limit imposed by the $s k y^{2}$, and the results completely fulfilled expectations, and even exceeded them in many respect, in particular regarding the quality of results concerning the polarisation of the CMB anisotropies.

The core cosmological result from Planck can be summarised concisely: a simple, six parameters, model $(\Lambda C D M)$ fits the data extremely well. This a rather extreme example of data compression, where trillions of bits of data are compressed to billions of sky measurements at 9 frequencies, then tens of millions of harmonic modes are compressed to thousands of binned multipoles which are in turn compressed to 6 cosmological parameters and a model. With no evidence for the need of a $7^{\text {th }}$, i.e., no indication that new physics (w.r.t. $\Lambda \mathrm{CDM}$ ) is needed! And this model is consistent with most other cosmological probes, but for "tensions" to which we shall return briefly.

Planck determined all of the parameters of this "base model", on its own, with exceptional accuracy. If we include polarization, the best determined parameter is know at the $0.03 \%$ level. Only one parameter is not determined to better than $1 \%$, i.e., the optical depth to reionization which cannot currently be determined from first principles and is therefore introduced as a nuisance parameter describing the effect of the end of the dark ages when the first collapsed objects reionized the Universe (and erased fluctuations at small scales and created new large scales ones).

Before proceeding any further, I want to stress that this 6 parameters (base) $\Lambda \mathrm{CDM}$ model is an amazingly minimal (effective) model, deceptively simple, since it relies on far reaching assumptions:

1. Physics is the same throughout the observable Universe.

2. General Relativity (GR) is an adequate description of gravity.

3. On large scales the Universe is statistically the same everywhere.

\footnotetext{
${ }^{1}$ Planck (http://www.esa.int/Planck) is a project of the European Space Agency (ESA) with instruments provided by two scientific consortia funded by ESA member states and led by Principal Investigators from France (JeanLoup Puget and myself) and Italy (Nazzareno Mandolesi and Marco Bersanelli), telescope reflectors provided through a collaboration between ESA and a scientific consortium led and funded by Denmark, and additional contributions from NASA (USA).

${ }^{2}$ The requirements for this "ultimate" measurement of the Cosmic Microwave Background (CMB) temperature anisotropies were then (i) full sky coverage \& angular resolution to survey all scales at which the CMB primary anisotropies contain information $\left(\sim 5^{\prime}\right)$ (ii) with a sensitivity essentially limited by the ability to remove the astrophysical foregrounds, i.e., with enough sensitivity within a large frequency range $[30 \mathrm{GHz}, 1 \mathrm{THz}]$. In polarisation, the goal was "only" to get the best performances possible on the polarization with the technology available at the time.
} 

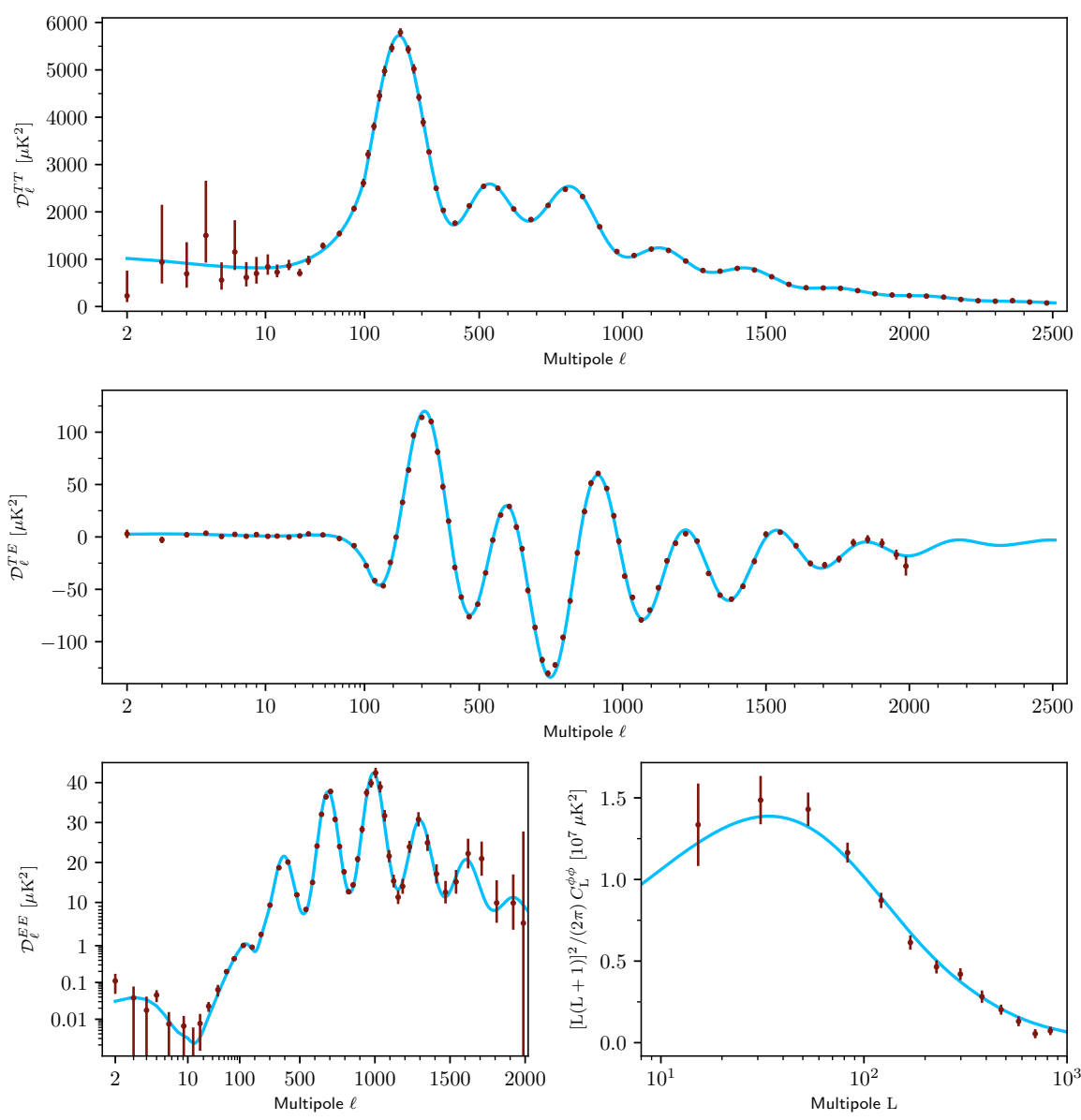

Figure 1: Planck 2018 CMB power spectra (binned) of TT (top), TE (middle), EE (bottom left), and $\Phi \Phi$ (bottom right) compared with the base $\Lambda$ CDM fit (blue line). T, E, $\Phi$ stand for temperature, E-mode polarisation and the LSS gravitational potential (projected along the line-of-sight; see text for details). For all plots, the horizontal scale changes from logarithmic to linear at the hybridization scale, $\ell=29$ (with a transition till $\ell=100$ ). (Reproduction of Fig. 9 of [3].)

4. The Universe was once much hotter and denser and has been expanding since early times.

5. There are five basic cosmological constituents:

(a) Dark energy that behaves just like the energy density of the vacuum.

(b) Dark matter that is pressureless (for the purposes of forming structure), stable and interacts with normal matter only gravitationally.

(c) Regular atomic matter that behaves just like it does on Earth.

(d) The photons we observe as the CMB.

(e) Neutrinos that are almost massless (again for structure formation) and stream like noninteracting, relativistic particles at the time of recombination.

6. The curvature of space is very small, dynamically negligible.

7. Variations in density were laid down everywhere at early times, and are Gaussian, adiabatic, and nearly scale invariant (i.e., proportionally in all constituents and with similar amplitudes as a function of scale), as predicted by inflation. 
8. The observable Universe has "trivial” topology (i.e., like R3).

All assumptions which Planck helped putting on a quite firm ground by checking for extensions or deviations. .. in addition to measuring precisely the corresponding minimal parameter set.

\section{CMB Consistency within $\Lambda$ CDM}

The CMB angular power spectra contain all of the information available if the CMB is statistically isotropic and distributed as a multivariate Gaussian, which we now know is an excellent approximation [4]. Throughout this paper, we refer to temperature, E and B-mode polarisation or lensing potential information ${ }^{3}$ with, respectively, the $T, E, B$, and $\Phi$ subscripts. The Planck papers $[5,6]$ obtain the $C_{\ell}^{T T}, C_{\ell}^{E E}, C_{\ell}^{\Phi \Phi}$ auto-spectra as well as the cross-correlation spectrum, $C_{\ell}^{T E}$, and $[7,8]$ detail the implications for the cosmological model and inflation.

Planck EE+lowE+BAO Planck TE+lowE Planck TT+lowE

Planck TT, TE,EE+lowE

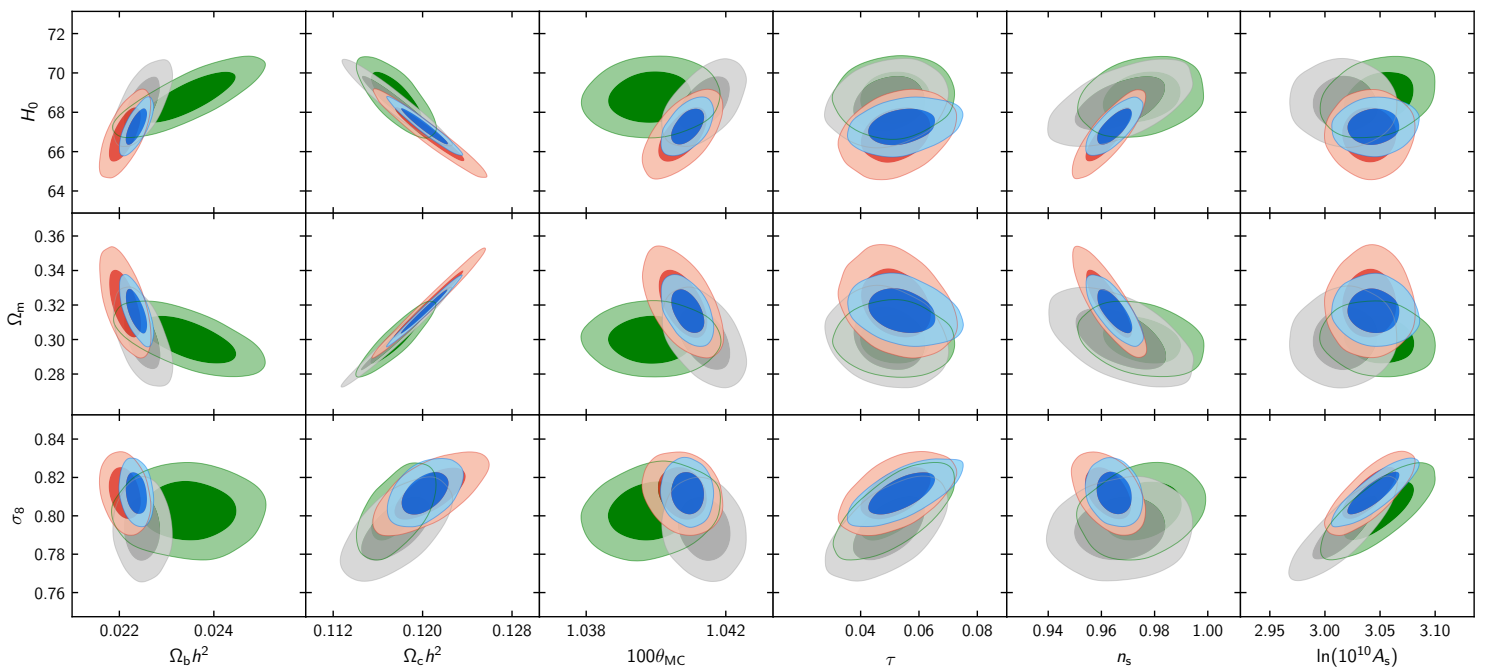

Figure 2: Constraints on parameters of the base- $\Lambda$ CDM model from the separate Planck EE, TE, and TT high $\ell$ spectra combined with low $\ell$ polarization data (lowE), and in the case of EE also with BAO, compared to the joint result using Planck TT, TE, EE+lowE. Parameters on the bottom axis are our sampled MCMC parameters with flat priors, and parameters on the left axis are derived parameters (assuming $\Lambda \mathrm{CDM}$ ), with $H_{0}$ in $\mathrm{km} \mathrm{s}^{-1} \mathrm{Mpc}^{-1}$. Contours contain $68 \%$ and $95 \%$ of the probability. (Reproduction of Fig. 5 of [7].)

Fig. 1 displays all these Planck spectra, compared with their best fit $\Lambda \mathrm{CDM}$ model, which was found to be an excellent statistical description of all the data. It is clearly seen that Planck detected and determined 7 acoustic peaks in TT, 6 in TE, and 5 in EE (to be compared with the $3 \& 2$ in TT\&TE of WMAP final release). It is quite remarkable that the quality of the data is now such that

\footnotetext{
${ }^{3}$ The deviations of CMB photons by LSS through their corresponding metric perturbations induce cross-correlations between CMB angular scales. One can then take advantage of these specific cross-correlations to reconstruct a map of the gravitational lensing potential $\Phi$ (of CMB photons by LSS), by an estimator which is essentially quadratic in the anisotropy fields. The lensing spectrum, $C_{\ell}^{\Phi \Phi}$ is therefore a specific four-point function, which complements the information in the other two-point functions. This lensing power spectrum therefore offers another check of consistency of the model, and since it arises at low- $z$, it allows breaking some degeneracies between models with different low- $z$ behaviours to which the primordial CMB is otherwise insensitive to.
} 
the temperature anisotropies, the E-mode anisotropies, or their correlation, each allow separately high precision determination of the $\Lambda \mathrm{CDM}$ parameters, to a level which till recently was only a dream for any combination of probes. This high-precision consistency is illustrated in Fig. 2. It is worth noting that the cross-correlation is nearly as powerful as the temperature part, and that the lensing degeneracy directions are actually different form the primordial CMB ones, which helps tightening further the full Planck data combination Planck TT, TE, EE+lowE.

CMB constraints from Planck alone and in combination with $\mathrm{BAO}$ are given in Table 1. The table shows that indeed all fundamental parameters of the base $\Lambda \mathrm{CDM}$ model (i.e., apart from the nuisance parameter, $\tau$ ) are determined at the per-cent level, with the angular scale of the sound horizon, $\theta$ (which determines the peaks spacing) being determined at the $0.03 \%$ level. These early Universe determinations allow predicting the values of a wide variety of derived parameters of physical interest, like the amplitude of fluctuation or the expansion rate today, $\sigma_{8}$ and $H_{0}$, assuming $\Lambda \mathrm{CDM}$ is correct. Some of these values are also given in the Table. We will return to this later when discussing tensions.

There are a number of well-motivated extensions to the base $\Lambda$ CDM model whose detection would provide essential clues on physics beyond the standard model of particle physics. Conversely, upper limits allow restricting the range of acceptable models. Regarding primordial physics, any detection of a deviation from a pure power-law primordial power spectrum of slope $n_{\mathrm{s}}$ (e.g., a non-zero "running", $\left.d n_{\mathrm{s}} / d \ln k\right)$, or the detection of a tensor component, $r=A_{\mathrm{t}} / A_{\mathrm{s}}$, of an isocurvature component, $\alpha_{-1}$, or of primordial non-Gaussianity of any kind, would be a complete game-changer. The CMB

\begin{tabular}{|c|c|c|}
\hline Parameter & Planck alone & Planck + BAO \\
\hline$\Omega_{\mathrm{b}} h^{2}$ & $0.02237 \pm 0.00015$ & $0.02242 \pm 0.00014$ \\
\hline$\Omega_{\mathrm{c}} h^{2}$ & $0.1200 \pm 0.0012$ & $0.11933 \pm 0.00091$ \\
\hline $100 \theta_{\mathrm{MC}}$ & $1.04092 \pm 0.00031$ & $1.04101 \pm 0.00029$ \\
\hline$\ldots \ldots$ & $0.0544 \pm 0.0073$ & $0.0561 \pm 0.0071$ \\
\hline $\ln \left(10^{10} A_{\mathrm{s}}\right) \ldots \ldots$ & $3.044 \pm 0.014$ & $3.047 \pm 0.014$ \\
\hline$\ldots \ldots \ldots$ & $0.9649 \pm 0.0042$ & $0.9665 \pm 0.0038$ \\
\hline$\overline{H_{0} \ldots \ldots \ldots \ldots}$ & $67.36 \pm 0.54$ & $67.66 \pm 0.42$ \\
\hline$\Omega_{\Lambda} \ldots \ldots \ldots$ & $0.6847 \pm 0.0073$ & $0.6889 \pm 0.0056$ \\
\hline$\Omega_{\mathrm{m}} \ldots \ldots \ldots$ & $0.3153 \pm 0.0073$ & $0.3111 \pm 0.0056$ \\
\hline$\Omega_{\mathrm{m}} h^{2} \ldots \ldots \ldots$ & $0.1430 \pm 0.0011$ & $0.14240 \pm 0.00087$ \\
\hline$\Omega_{\mathrm{m}} h^{3}$ & $0.09633 \pm 0.00030$ & $0.09635 \pm 0.00030$ \\
\hline$\sigma_{8} \ldots \ldots \ldots \ldots$ & $0.8111 \pm 0.0060$ & $0.8102 \pm 0.0060$ \\
\hline$\sigma_{8}\left(\Omega_{\mathrm{m}} / 0.3\right)^{0.5}$ & $0.832 \pm 0.013$ & $0.825 \pm 0.011$ \\
\hline$z_{\text {re }}$ & $7.67 \pm 0.73$ & $7.82 \pm 0.71$ \\
\hline Age $[\mathrm{Gyr}] \ldots \ldots$ & $13.797 \pm 0.023$ & $13.787 \pm 0.020$ \\
\hline$r_{*}[\mathrm{Mpc}] \ldots \ldots$ & $144.43 \pm 0.26$ & $144.57 \pm 0.22$ \\
\hline $100 \theta_{*}$ & $1.04110 \pm 0.00031$ & $1.04119 \pm 0.00029$ \\
\hline$r_{\mathrm{drag}}[\mathrm{Mpc}] \ldots \ldots$ & $147.09 \pm 0.26$ & $147.57 \pm 0.22$ \\
\hline$z_{\mathrm{eq}} \ldots \ldots \ldots$ & $3402 \pm 26$ & $3387 \pm 21$ \\
\hline$k_{\mathrm{eq}}\left[\mathrm{Mpc}^{-1}\right]$ & $0.010384 \pm 0.000081$ & $0.010339 \pm 0.000063$ \\
\hline$\Omega_{K} \ldots \ldots \ldots \ldots$ & $-0.0096 \pm 0.0061$ & $0.0007 \pm 0.0019$ \\
\hline$\Sigma m_{v}[\mathrm{eV}] \ldots \ldots$ & $<0.241$ & $<0.120$ \\
\hline$N_{\text {eff }}$ & $2.89_{-0.38}^{+0.36}$ & $2.99_{-0.33}^{+0.34}$ \\
\hline$r_{0.002}$ & $<0.101$ & $<0.106$ \\
\hline$w_{0} \ldots \ldots \ldots$ & $-1.57_{-0.40}^{+0.50}$ & $-1.04_{-0.10}^{+0.10}$ \\
\hline
\end{tabular}

Table 1: Parameter confidence limits from Planck CMB temperature, polarization and lensing power spectra, alone and with the inclusion of BAO data. The first set of rows gives $68 \%$ limits for the base- $\Lambda \mathrm{CDM}$ model, while the second set gives $68 \%$ constraints on some derived parameters (as obtained from the constraints on the parameters used to specify the base- $\Lambda \mathrm{CDM}$ model). The third set below the double line gives $95 \%$ limits for some 1-parameter extensions to the $\Lambda \mathrm{CDM}$ model. anisotropies could also reveal deviations from the minimal assumptions behind the base $\Lambda$ CDM model. The CMB anisotropies could in particular allow detecting spatial hypersurfaces departing from flat, $\Omega_{K}=1-\Omega_{\mathrm{m}}-\Omega_{\Lambda} \neq 0$, a non-minimal "Dark energy" equation of state, $w \neq-1$, the sum of the neutrinos masses, $\sum m_{v}$, being greater than their lower limit $\simeq 0.06 \mathrm{eV}$ from flavour oscil- 


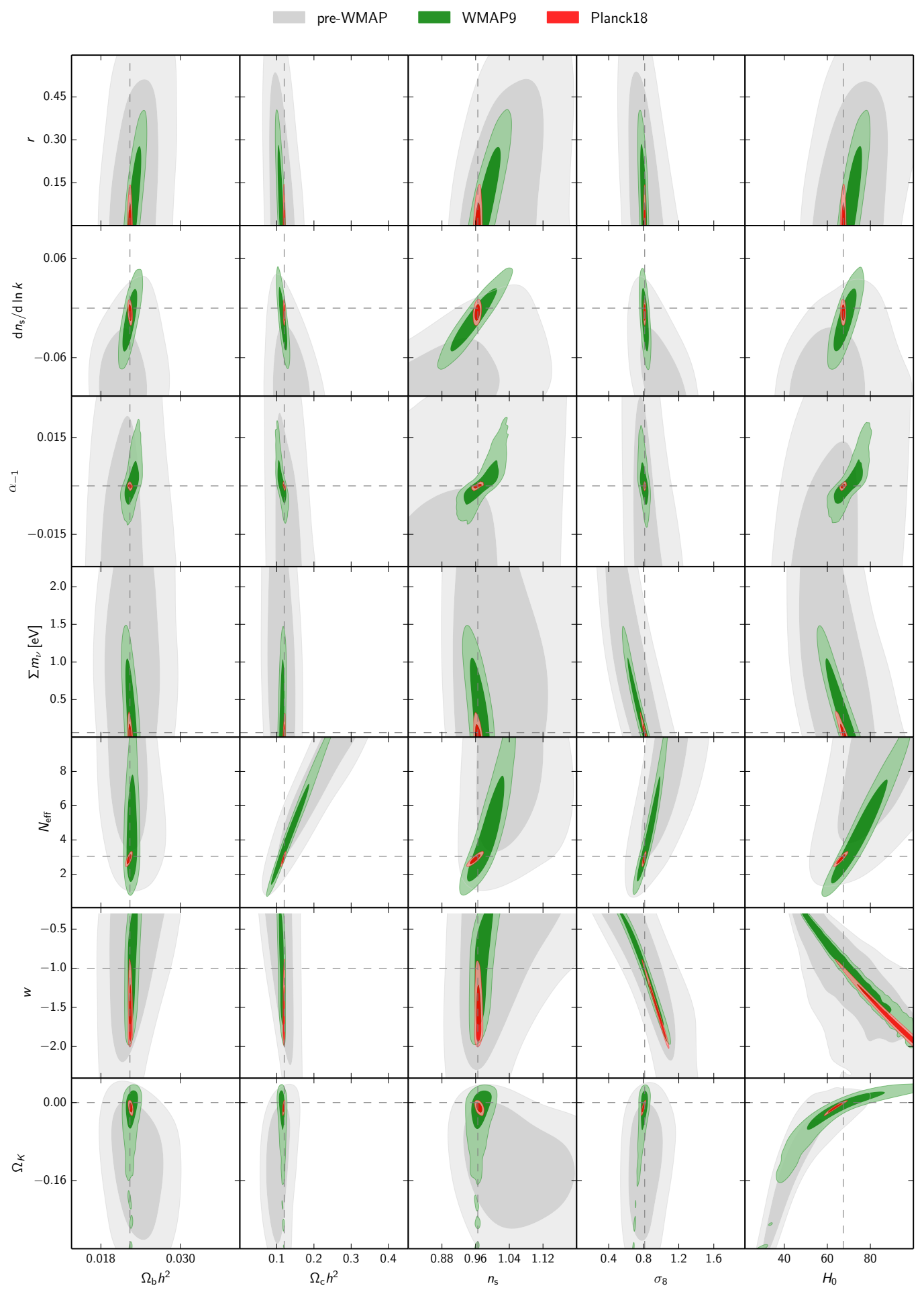

Figure 3: Successive reductions in the allowed parameter space for various one-parameter extensions to $\Lambda C D M$, from pre-WMAP (the MAXIMA, DASI, BOOMERANG, VSA, and DASI experiments) to Planck. Each row corresponds to a specific extended model. The contours display the $68 \%$ and $95 \%$ confidence limits for the extra parameter versus five other base- $\Lambda \mathrm{CDM}$ parameters. The dotted lines indicate the $\Lambda \mathrm{CDM}$ best-fit parameters or fixed default values of the extended parameters. (Reproduction of Fig. 14 of [3].) 
lation experiments, or the existence of extra (light) degrees of freedom, their weighted sum $N_{\text {eff }}$ being then different from its canonical value of $\simeq 3.046$. And many more, like the existence of topological defects, a variation of the fundamental constants, or deviations from General Relativity... Planck does not require any of these extensions, and Table 1 provides for easy reference some of these current upper-limits, including those from Planck+BAO that lifts some low- $z$ degeneracies (last column). These are for most of them the best available.

One of the achievement of current CMB data is that it has now reached a level such that opening of these additional degrees of freedom does not jeopardize any more the constraints on the base parameters. One may for instance examine in Fig. 3 the constraints on $n_{\mathrm{s}}$ at different stages of development of the CMB field. Before Planck, the deviation from scale invariance, $n_{\mathrm{s}}<1$, expected from inflation was certainly not robust against many reasonable extensions (and in particular $r$, $d n_{\mathrm{s}} / d \ln k, \alpha_{-1}$, or $N_{\text {eff }}$ ). With Planck, $n_{\mathrm{s}}$ remains always at least $3 \sigma$ away from one (instead of $8 \sigma$ in the base $\Lambda$ CDM case). It is also worth noting that $H_{0}$ constraints were also very dependent on these untested assumptions. Finally, this figure also shows how much more constrained these extensions are. This all results in a massive reduction of the allowed space of models.

We shall see below (Fig. 5) that this trend will continue, and note for now that the other CMB experiments, while completely consistent with Planck, have not yet reached the stage where there inclusions would improve significantly upon Planck constraints, but for the upper limit on the amplitude of primordial gravitational waves (where Planck alone, indirect constraint, $r<0.10$, improves to $r<0.06$ at 95\% C.L. when the latest B-mode results from Bicep-Keck are considered).

\section{Cosmological Consistency within $\Lambda \mathrm{CDM}$}

The tight agreement between the observational determination of the primordial Helium and Deuterium abundances, the calculation of Big Bang Nucleosynthesis, and the constraint on the

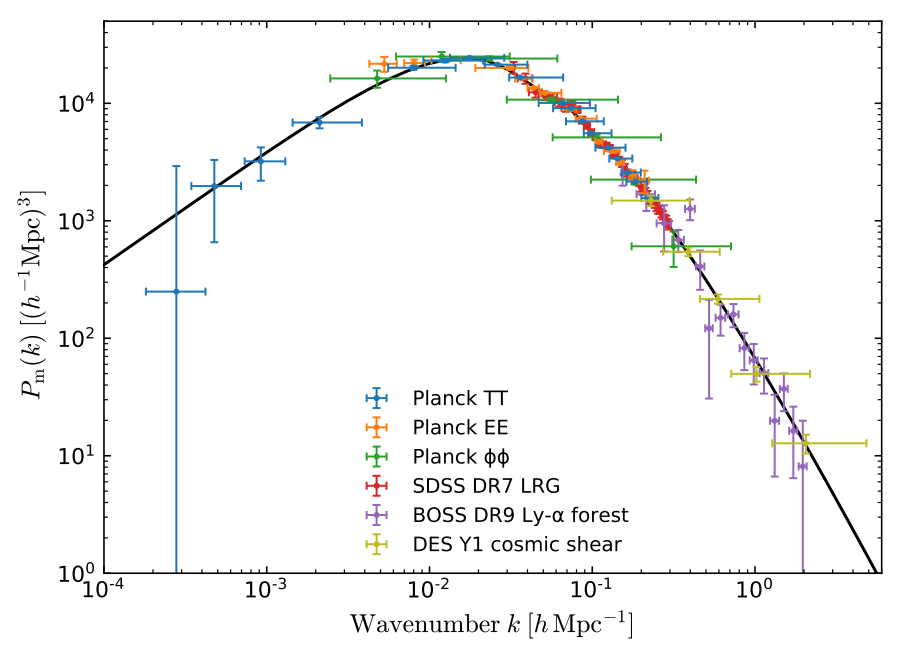

Figure 4: The (linear theory) matter power spectrum (at $z=0$ ) inferred from different cosmological probes. (Reproduction of Fig. 19 of [3].) 
baryonic abundance from Planck, is a strong indication that physics is universal. Indeed this comparison uses all known laws of physics, across aeons!

Concerning the late Universe probes, the inferences from $\Lambda \mathrm{CDM}$ with parameters determined by Planck are in good agreement with BAO data (on the acoustic scale $D / r_{\text {drag }}$ ), with redshift space distortions which probe the growth rate of structures $\left(f=d \ln D / \ln a \sim \omega_{\mathrm{m}}^{0.55}\right.$ in $\left.\Lambda \mathrm{CDM}\right)$, with relative distances of supernovae and with weak lensing distortions of background galaxies. These low-redshift probes can then be used to better break residual CMB degeneracies (i.e., the CMB is only sensitive to the low- $z$ universe through lensing and angular scales), in particular on $w$.

One way to graphically see this impressive data consistency within $\Lambda C D M$ is given in Fig. 4. This figure shows the (linear theory) matter power spectrum (at $z=0$ ) inferred from different cosmological probes. The broad agreement of the model (black line) with such a disparate compilation of data, spanning $14 \mathrm{Gyr}$ in time and three decades in scale is an impressive testament to the explanatory power of $\Lambda \mathrm{CDM}$.

Still there are a number of tensions whose meaning at this time is not clear yet. There are mild tensions with weak lensing surveys once galaxy clustering constraints are added to pure lensing ones. More significantly, there is a strong, $4.4 \sigma$, tension with the local measurement of $H_{0}$ when using Supernovae calibrated/anchored with, e.g., Cepheids (there is no tension using the inverse distance ladder where Supernovae relative distances are anchored with BAO and CMB or $\mathrm{BBN})$. While a number of systematics effects potentially affecting the local determination have been pointed out, there is currently no consensus on that point, apart from the need to continue the analyses, decrease the uncertainties of the local measurements, and bring to bear, to the extent possible, completely new ways to measure $H_{0}$. This is all of paramount importance since it may signal the detection of new physics... or so far unaccounted or unknown systematics.

\section{Future prospects and Conclusions}

One point I wish to stress is what makes the CMB so special for Cosmology. The CMB can be accurately measured, compared to precise theoretical predictions, with a rich phenomenology, in a statistically reliable, and computationally tractable way. There are very few situations in cosmology, astrophysics (or indeed physics) where all of these conditions are met. It is the intersection of these qualities that makes CMB such a powerful cosmological probe! And this gold mine is nowhere near to be exhausted.

Fig. 5 displays the successive reductions of the parameter space allowed by the data ${ }^{4}$, as measured by the figure of merit FoM $=\left\{\operatorname{det}\left[\operatorname{Cov}\left(\Omega_{\mathrm{b}} h^{2} ; \Omega_{\mathrm{c}} h^{2} ; \tau, A_{\mathrm{s}} ; n_{\mathrm{s}} ; \ldots\right)\right]\right\}^{-1 / 2}$, where Cov stands for the covariance matrix of the cosmological parameters considered. This is done for various models and data sets, relative to COBE (for which we have additionally (anachronistically) assumed a Planck prior on the optical depth $0.055 \pm 0.009$ ). The relative reduction of the allowed phase space volume is impressive for all models, with even greater shrinkage in volume for higher-dimensional model extensions. For $\Lambda \mathrm{CDM}$, the Planck improvement versus COBE is more than $10^{10}$. For the largest model spaces, having four or five additional dimensions compared to $\Lambda \mathrm{CDM}$, this improvement is more than $10^{16}$ in 26 years, corresponding to a sustained 6 month doubling time (3 times

\footnotetext{
${ }^{4}$ Indeed, this is inversely proportional to the (square root of) the product of the variances of the parameter combinations for which the Covariance matrix is diagonal
} 
shorter than for Moore's Law!). This is one reason why the study of the CMB has allowed us to address more and more ambitious questions with time, with subtle effects revealing the imprint of more physics as the experiments become more capable. As the figure shows, this is expected to continue with future $\mathrm{CMB}$ experiments, extending further our capability to find limitations, or even failure, of the standard model of cosmology (even if the current tensions are resolved).

The current breed of CMB experiments on the ground (e.g., ACT, BICEP/Keck, CLASS, Polarbear/Simons Array, Simons Observatory, SPT), which in the USA is collectively referred to as Stage 3, or CMB-S3, deploys of the order of $\lesssim 20000$ detectors per experiment. They pave the way to the next generation experiment, dubbed CMB-S4, which will pass quite significant scientific threshold in the pursuit of inflation, neutrino properties, dark energy, and much more [9]. It calls for $\sim 500000$ detectors spanning $20-270 \mathrm{GHz}$ using multiple telescopes and sites to map most of the sky, as well as deep targeted fields. It involves a broad participation of the CMB community, including existing CMB-S3 experiments, National Laboratories, and the High Energy Physics community. It is a joint project between NSF (AST, PHY, OPP) and DOE (HEP), with an estimated cost of $\sim 600 \mathrm{M} \$$ till the start of operations in FY2027. International partnerships is encouraged, but there is (yet?) no joint European response to this offer.

On the same time-scale than CMB-S4, there is the JAXA-led satellite project called LiteBIRD, which should also be launched in 2027. The full success requirement of this more narrowly targeted mission is a measurement of the tensor-to-scalar ratio, $r$, at $\sigma(r)<0.001$ (without delensing), i.e., in the same ballpark than the $\mathrm{S} 4$ target for $r$. This is to be achieved by measuring the B-mode polarisation spectrum at large angular scales $(2<\ell<200)$ with 3 years of surveying; this will then also measure $\tau$ exquisitely. While liteBIRD will be competing with $\mathrm{S} 4$ on a tentative detection of the primordial gravitational wave background, there are also obvious synergies, since Litebird covers the full sky, and includes frequencies unattainable from the ground $(40-400 \mathrm{GHz}$ in 15 frequency bands, which may turn out to be necessary for a solid detection claim). In addition, both large and small(er) scales measurements will be required to reach the best determination of

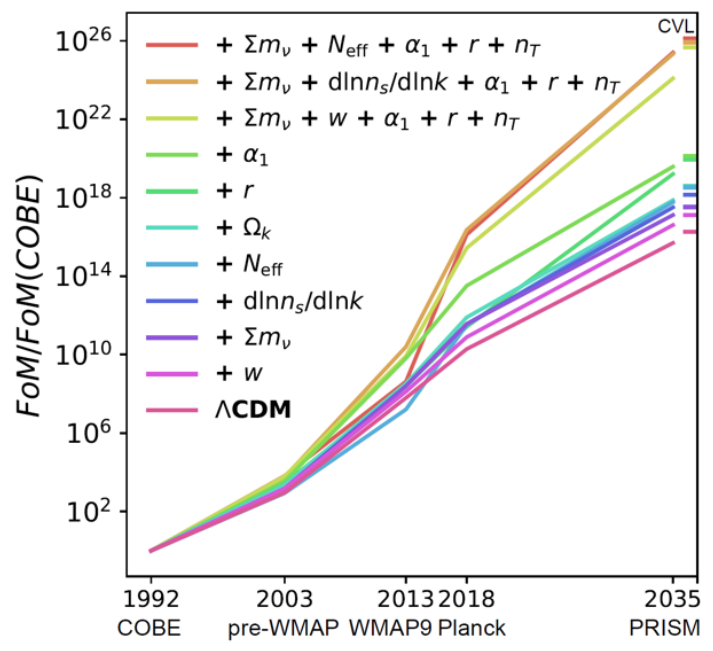

Figure 5: Successive improvements in the constraining power (FOM relative to COBE, see text) of various experiments for various models. (Reproduction of Fig. 19 of [3], updated for the PRISM proposal). The "CVL" ticks at right give the cosmic variance limit, for a perfect noise-free, all-sky experiment. 
the lensing potential (which calls for measuring lensing-induced correlations over a broad range of scales). Note that determining the lensing potential not only helps in measuring lower levels of primordial B-modes, it has also tremendous scientific potential of its own, from high-z $(\sim 2)$ constraints on modified gravity theories, to breaking degeneracies in the light-to-mass ratio plaguing cosmological studies of galaxy formation, or measuring the sum of neutrinos mass (which sets when they become non-relativistic, i.e., the scale below which fluctuations have been damped by free-streaming).

In the longer term, we shall also revisit the COBE/FIRAS upper limits on spectral distortions, ultimately targeting the inescapable imprint of the "Silk" damping of acoustic waves due to the imperfect nature of the pre-recombination baryon-photon fluid. This will give us constraints on the primordial curvature power spectrum at smaller scales than possible with the CMB anisotropies, in addition to allowing a detection of a possible non-standard thermal history (e.g., due to unexpected particles, or interactions). Even more demanding will be the detection of recombination lines. We shall also fully use the scientific potential of the CMB as a back light with well-understood properties, to measure warm baryons, large-scale velocity fields, resolve the cosmic infrared background, etc. This will require further progress on achievable sensitivity and large telescopes to access smaller scales.

The next decade will also be rich in new Large Scale Structure surveys which will probe the low(er)- $z$ Universe with many different probes. In particular, Baryon Acoustic Oscillations (BAO), Redshift Space Distortion (RSD), Supernovae (SNe), Weak Lensing (WL) and Galaxy Clustering (GC). Each survey and probe will have a different sensitivity to cosmology, nuisance parameters (mass-to-light biases)..., and systematics! Let me mention a few of these experiment with in () the probes they will employ and in [] their planned starting date of operations: DESI (BAO, RSD) [2019], LSST (WL, SNe, BAO w/ photo-z, GC) [2022], Euclid (WL, BAO, RSD, GC) [2022], SPHEREx (GC) [2023], WFIRST (WL, BAO, RSD, SNe, GC) [2025], SKA1 (Intensity Mapping) [2027].

Not only will this profusion of probes guarantee progress and discoveries on many different fronts (including the challenging formation of galaxies and clusters) and maybe find evidence for new physics, but it also may (and I am tempted to say will) reveal systematics or oversimplified theoretical models. In any case, this multiplicity of approaches and relations to the underlying cosmological model will enable a robust physical interpretation of the data. And there will be work for many, in particular on the theory side, since the full potential of the low- $z$ data often lies at small, strongly non-linear, scales which most often involve complicated physics further to gravity. Very large scale analysis will also require full GR calculations (as imposed to the Newtonian limit which is most often sufficient for current LSS studies). And last but not least, this deluge of data will require novel data analysis approaches. In any case, the whole of these data will be much greater than the sum of its parts, "cosmogold" will keep coming, and it will continue to be fun and exciting to be a cosmologist!

\section{Acknowledgments}

A description of the Planck Collaboration and a list of its members, including the technical or scientific activities in which they have been involved, can be found at http://www. sciops. esa. int/index .php?project=planck\&page=Planck_Collaboration. 


\section{References}

[1] C. L. Bennett, D. Larson, J. L. Weiland, N. Jarosik, G. Hinshaw, N. Odegard et al., Nine-Year Wilkinson Microwave Anisotropy Probe (WMAP) Observations: Final Maps and Results, ArXiv e-prints (2012) [1212.5225].

[2] Planck Collaboration I, Planck early results. I. The Planck mission, AEA 536 (2011) A1 [1101.2022].

[3] Planck Collaboration I, Planck 2018 results. I. Overview, and the cosmological legacy of Planck, AESA, submitted (2018) [1807.06205].

[4] Planck Collaboration VII, Planck 2018 results. VII. Isotropy and statistics, AEAA, in press (2018) [1906.02552].

[5] Planck Collaboration V, Planck 2018 results. V. Power spectra and likelihoods, AEAA, accepted (2018) [1907.12875].

[6] Planck Collaboration VIII, Planck 2018 results. VIII. Gravitational lensing, AEAA, in press (2018) [1807.06210].

[7] Planck Collaboration VI, Planck 2018 results. VI. Cosmological parameters, AEA, submitted (2018) [1807.06209].

[8] Planck Collaboration X, Planck 2018 results. X. Constraints on inflation, AEA, submitted (2018) [1807.06211].

[9] K. N. Abazajian, P. Adshead, Z. Ahmed, S. W. Allen, D. Alonso, K. S. Arnold et al., CMB-S4 Science Book, First Edition, arXiv e-prints (2016) [1610.02743]. 